\title{
Die historischen Avantgarden in Lateinamerika
}

Vor einiger Zeit fand in Berlin eine von Harald Wentzlaff-Eggebert veranstaltete internationale Tagung mit dem Titel Europäische Avantgarde im lateinamerikanischen Kontext statt. ${ }^{1}$ Ihr Veranstalter, der auch in der Folge mehrere Workshops und Sektionen zu diesem Thema organisierte und zum damaligen Zeitpunkt als der in Deutschland wohl beste Kenner der Materie bezeichnet werden darf, hatte zweifellos gute Gründe dafür, von „europäischer Avantgarde“ zu sprechen. Dennoch stellen sich mit Bezug auf den Titel der Veranstaltung Fragen. Zweifellos kann man für weite Strecken noch des 19. Jahrhunderts behaupten, dass innerhalb einer zunehmend globalen Literaturentwicklung die Impulse aus Europa kamen und dann etwa in Lateinamerika aufgenommen, abgewandelt und transformiert wurden. Aber spätestens mit dem hispanoamerikanischen Modernismo war dies nicht mehr der Fall, ging dieser doch - verkürzt gesagt - von Spanisch-Amerika und nicht länger von Spanien aus und entwickelte sich dieser Modernismus ${ }^{2}$ doch zunehmend zu einer literarisch-ästhetischen Bewegung, welche keineswegs nur in Spanien, sondern weltweit ihre Impulse aus Lateinamerika erhielt.

Dies haben in den vergangenen Jahrzehnten die Forschungen längst aufgezeigt, die ich an gegebener Stelle in unserer Vorlesung zu den Romanischen Literaturen der Welt im 19. Jahrhundert auch bespreche. An dieser Stelle nur so viel: Der geokulturelle Dominantenwechsel, der sich mit der politischen Unabhängigkeit, der „Independencia“, in Hispanoamerika im literarischen Feld einstellte und über lange Zeiten die Romantik zwischen zwei Welten in ihrer Entwicklung bestimmte, veränderte sich mit dem hispanoamerikanischen Modernismus. Dies insoweit, als es zwar keinesfalls zu einer Independencia dieser Literaturen der spanischsprachigen Welt Amerikas gekommen wäre, wohl aber zur Neubestimmung einer Asymmetrie der Beziehungen, ${ }^{3}$ welche in diesem Falle zum ersten Mal den literarischen Ausgangsimpuls einer Bewegung in Lateinamerika verortete. Sollte nun all dies mit den historischen Avantgarden Lateinamerikas

1 Vgl. die Akten dieser Tagung in Wentzlaff-Eggebert, Harald: Europäische Avantgarde im lateinamerikanischen Kontext. La Vanguardia europea en el contexto latinoamericano. Actas del Coloquio Internacional de Berlín 1989. Frankfurt am Main: Vervuert 1991.

2 Vgl. auch Asholt, Wolfgang (Hg.): Avantgarde und Modernismus. Dezentrierung, Subversion und Transformation im literarisch-künstlerischen Feld. Berlin - Boston: Walter de Gryuter 2014.

3 Vgl. hierzu Ette, Ottmar: Asymmetrie der Beziehungen. Zehn Thesen zum Dialog der Literaturen Lateinamerikas und Europas. In: Scharlau, Birgit (Hg.): Lateinamerika denken. Kulturtheoretische Grenzgänge zwischen Moderne und Postmoderne. Tübingen: Gunter Narr Verlag 1994, S. 297-326; sowie Janik, Dieter: Hispanoamerikanische Literaturen. von der Unabhängigkeit bis zu den Avantgarden (1810-1930). Tübingen - Basel: Francke 2008.

Ә Open Access. (C) 2021 Ottmar Ette, publiziert von De Gruyter. (c) BB-NC-ND Dieses Werk ist lizensiert unter einer Creative Commons Namensnennung - Nicht-kommerziell - Keine Bearbeitung 4.0 International Lizenz. https://doi.org/10.1515/9783110703450-008 
in den status quo ante zurückgefallen sein? Oder pointierter: Empfing man in Lateinamerika wieder - wie über lange Jahrhunderte zuvor - alle künstlerischen und literarischen Impulse aus Europa und betätigte sich ,nur` in deren Variation und Weiterentwicklung? Saßen also in Lateinamerika lediglich die Rezipienten der historischen Avantgarde in Europa und konnten bestenfalls halbwegs kreativ darauf reagieren?

Lassen Sie uns nochmals anders fragen: War die Avantgarde in Lateinamerika lediglich ein Abklatsch jener ,europäischen Avantgarde‘, deren literarhistorische Entwicklung seit dem italienischen Futurismus wir verfolgt haben und die wir in Abgrenzung zu den späteren Neo-Avantgarden des 20. Jahrhunderts als die „historischen“ bezeichneten? Um auf all diese Fragen eine einfache Antwort zu geben, die zugleich auch das Movens unserer eigenen Untersuchung im Zeichen der TransArea Studies berührt: keineswegs! Denn die künstlerische Kreativität wie die spezifisch literarischen Eigenentwicklungen in Lateinamerika waren auf dem Gebiet der Ausbildung historischer Avantgarden immens.

Der zum damaligen Zeitpunkt am Lateinamerika-Institut der Freien Universität Berlin tätige Kolumbianer Carlos Rincón hat in seinem Beitrag zu der genannten Tagung ${ }^{4}$ zunächst auf die schwierige Gleichzeitigkeit von politischer und ästhetischer Avantgarde auch und gerade in Lateinamerika aufmerksam gemacht. Oftmals waren es ja die politischen Avantgarden, die auf ästhetisch-literarischem Gebiet eben nicht zur Vorhut zählten, sondern allenfalls die Nachhut bildeten und dabei keinesfalls das Versprechen des Wagnisses und des unbedingten Fortschrittswillens einlösten, das sie auf politischem Gebiet gegeben hatten. Rincón wandte sich im Übrigen auch strikt gegen alle nachträglichen Versuche von politisch interessierter Seite, die Positionen eines José Carlos Mariátegui, die von redlichen orthodoxen Kommunisten noch in den fünfziger Jahren als populistisch beschimpft worden waren, post festum als Versöhnung von politischer und ästhetisch-intellektueller Avantgarde zu beweihräuchern. Man könnte an dieser Position aus heutiger Sicht der Mariátegui-Forschung durchaus ein Fragezeichen anbringen und für eine versöhnlichere Haltung des Peruaners in seinen Siete ensayos optieren.

Die immer wieder angestrebte Verknüpfung von politischer mit literarischer Avantgarde hält freilich in Lateinamerika bis in die achtziger Jahre an und ist letztlich bis heute nicht völlig verschwunden. Die Metaphorik der Avantgarde selbst legt dies freilich nahe, versteht sie sich doch als Vorhut, die in ein in Feindeshand befindliches Territorium eindringt, dessen Eroberung sie vorbereitet.

4 Vgl. Rincón, Carlos: la vanguardia en Latinoamérica: posiciones y problemas. In: WentzlaffEggebert, Harald (Hg.): Europäische Avantgarde im lateinamerikanischen Kontext, S. 51-75. 
Metaphorisch ist die Avantgarde also an scharfer Opposition, an Gegnerschaft, an Kampf, Schlacht und tödlichen Begegnungen, aber auch an einer Vorwärtsmetaphorik ausgerichtet, welche auch die zeitliche Dimension in diesen Begriff einbringt. Denn es sind die Avantgardisten, die zu den ersten Kündern des Neuen gerade dort werden, wo derlei Neuerungen noch militant das Alte im Wege steht. Ziel aber ist zweifellos die Beherrschung des Feindeslandes und jene Ästhetik des Bruchs mit dem Alten, mit dem Feind, die in Peter Bürgers Theorie der Avantgarde - wie wir sahen - vorherrscht. Damit ist bekanntlich eine völlig andersgeartete Definition der Institution Kunst verbunden.

Der Frage, ob auch in Latein- beziehungsweise Hispanoamerika die Avantgarde für eine Ästhetik des Bruchs einsteht, werden wir uns noch stellen müssen, galt es doch auch in Bezug auf die Amerikas lange Zeit als ausgemacht, dass auch diese Avantgarden letztlich derselben, am europäischen Modell ausgerichteten Theorie gehorchen würden. Noch die chilenische Kulturtheoretikerin Ana Pizarro verstand in ihrem Projekt einer Sozialgeschichte der lateinamerikanischen Literatur die Avantgarde als Bruch mit der Tradition und im Sinne Bürgers als Infragestellung der Institution Kunst. Wir werden im Verlauf dieses Kapitels sehen, ob sich diese Theorie aufrecht erhalten lässt angesichts der von uns ins Auge gefassten spezifischen Bedingungen des Schreibens in Lateinamerika. Denn die Asymmetrie der Beziehungen zwischen Europa und Amerika ist erhalten geblieben, nur hat sie sich spätestens seit dem Modernismo grundlegend verändert.

In einem thesenartigen Aufriss hat Harald Wentzlaff-Eggebert versucht, einige Charakteristika der hispanoamerikanischen Avantgarde, die in Europa bislang nur ungenügend aufgearbeitet worden sei, zu präsentieren und darzustellen. ${ }^{5}$ Dabei betonte er, dass die Avantgarde nicht nur ein Phänomen der Literatur, sondern der verschiedenen Künste, insbesondere auch Film und Malerei, in Lateinamerika wie in Europa gewesen sei. Wir dürfen unsererseits daraus die Notwendigkeit einer inter- und transmedialen Sichtweise, welche die Verbindung zwischen den verschiedenen Künsten betont, hervorheben. Innerhalb der Literatur sah Wentzlaff-Eggebert die Schwerpunkte avantgardistischer Tätigkeit im Bereich der Lyrik, aber auch des lateinamerikanischen AvantgardeTheaters. Wir wollen versuchen, durch unsere vorgeschaltete Untersuchung eines Versdramas des Mexikaners Alfonso Reyes beide Bereiche, Lyrik und Theater, in unserer Analyse miteinander zu verbinden.

Die Avantgardebewegungen kommen in Lateinamerika unverkennbar aus den großen Metropolen des Kontinents und werden von den gebildeten wohl-

5 Vgl. Wentzlaff-Eggebert, Harald: Sieben Fragen und sieben vorläufige Antworten zur Avantgarde in Lateinamerika. In: Iberoromania (Tübingen) 33 (1991), S. 15-139. 
habenden Schichten und insbesondere deren Sprösslingen getragen. Ihre großen Zeitschriften versuchen, nicht nur die regionalen Sonderentwicklungen, sondern ganz Lateinamerika in ihre Berichte und Texte miteinzubeziehen: Erinnert sei hier nur an die Revista de Avance in Kuba, deren fünfzig Nummern zwischen 1927 und 1930 erschienen, oder an die peruanische Revista Amauta, die keineswegs bloß regionale Phänomene darstellten. ${ }^{6}$ Die Neigung zu Manifesten, die die historischen Avantgarden insgesamt auszeichnen, schlug sich auch in diesen Zeitschriften nieder.

Zweifellos waren diese Avantgarden, wie sich etwa anhand der kubanischen Minoristen zeigen ließe, sehr stark an Europa und insbesondere an Phänomenen wie dem italienischen Futurismus, dem spanischen Ultraísmus oder dem französischen Surrealismus orientiert. ${ }^{7}$ Deren Entwicklungen vollzogen sie stets begierig und rasch nach innerhalb einer Welt, die sich nicht nur im wirtschaftlichen, sondern auch im kulturellen Bereich immer stärker vernetzte. Die Avantgarden in Lateinamerika zeichnet dabei ein deutlich politischer Charakter aus, wurde doch jeder Inkonformismus sogleich in diesem Sinne gedeutet. Angriffsziele bildeten im spezifisch literarischen Bereich bestimmte Schreibweisen und elegante Preziosismen des Modernismo, im politischen Bereich aber auch die pathetisch politische Rede, wie sie selbst die Modernisten - denken wir nur an den Kubaner José Martí oder den Uruguayer José Enrique Rodó - gepflegt hatten.

So etablieren sich die verschiedenen avantgardistischen Diskurse laut Harald Wentzlaff-Eggebert als Anti-Diskurse, eine Aussage, an der ich freilich gerade bezüglich jener Kontinuitäten, die ich im Folgenden aufzeigen möchte, gewisse Zweifel hege. Wir werden im Verlauf unserer Vorlesung derlei Fragen gehörig auf den Leib rücken und sie diskutieren.

Eines jedoch ist sicher: Die Avantgarde lässt sich zweifellos auch in Lateinamerika nicht als bloßer Epochenstil abtun, unternimmt sie doch erfolgreich den Versuch, ganze Diskurssysteme in Frage zu stellen oder zu überwinden und zugleich die Grenzen der Literatur selbst in den Bereich des Alltags hinauszuschieben. In dieser Hinsicht ist die Entwicklung in Lateinamerika durchaus vergleichbar mit den verschiedenen avantgardistischen Bewegungen in Europa. Die mexikanische wie die russische Revolution prägten vom zweiten Jahrzehnt des

6 Vgl. González, Alexandra Pita: La Unión Latinoamericana y el Boletín Renovación. Redes intrnacionales y revistas culturales de la década de 1920. México - Colima: El Colegio de México Universidad de Colima 2009.

$7 \mathrm{Zu}$ den Besonderheiten der spanischen Avantgarde und deren Transzendierung vgl. Buschmann, Albrecht: Max Aub und die spanische Literatur zwischen Avantgarde und Exil. Berlin Boston: Walter de Gruyter 2012. 
zwanzigsten Jahrhunderts an die grundlegend politisch wirkenden avantgardistischen Überlegungen, Manifeste und Manifestationen in Lateinamerika. Doch ließe sich dies auch von den Avantgarden Europas sagen.

Was also sind die Spezifika avantgardistischen Schreibens in der Neuen Welt? Gewiss, da ist die unzweifelhaft deutlichere Politisierung, ja Einbindung in politische Revolutionen zu beachten, was zweifellos mit der erst beginnenden relativen Autonomisierung des literarischen Feldes in den verschiedenen lateinamerikanischen Ländern zu tun hat. Doch die Beantwortung der Frage macht einen Rekurs auf die grundsätzlich verschiedenen gesellschaftlichen, sozioökonomischen, literarhistorischen und ästhetischen Bedingungen der Avantgarde in Lateinamerika unausweichlich. Wir wollen die Beantwortung dieser so komplexen Thematik folglich in verschiedenen Etappen unternehmen.

Ohne jede Frage ist es so, dass eine Avantgarde in Lateinamerika ohne die Entwicklung der europäischen Avantgarden nicht denkbar war. Der italienische Futurismus ab 1909, mit seiner Technikeuphorie noch vor dem Ersten Weltkrieg, war ein Fanal, das nur wenige Wochen nach dem Erscheinen des Gründungsmanifests in Le Figaro von keinem Geringeren als dem Modernisten Rubén Darío im damaligen Buenos Aires aufgenommen und verbreitet wurde. Dies war zweifellos eine Initialzündung aus Europa, die fast sofort auf Grundlage der schnellen transozeanischen Kommunikationsmittel aufgenommen wurde und künstlerischliterarisch wirkte. Dabei ist es eine ebenso schöne wie aussagekräftige Anekdote, dass es der damals führende Kopf der „Modernistas“, der nikaraguanische Dichterfürst und Essayist war, der die Gedanken Marinettis als erster in Lateinamerika verbreitete und damit einer Avantgarde auf die Sprünge half, von der bis heute behauptet wird, dass sie die unerbittliche Gegnerin der Modernisten gewesen sei. Es ist einfach, mittels derlei simpler Behauptungen eine bloße Übertragung europäischer Vorstellungen nach Lateinamerika zu erblicken.

Aber auch die sich anschließenden Bewegungen fanden rasch fruchtbare Aufnahme. So etwa der Dadaismus während des Ersten Weltkriegs ab 1916 mit seiner klar a-mimetischen Haltung, gerichtet gegen bürgerlich-nationale Konstrukte, die Europa ja gerade in den Krieg gezogen hatten - unvergesslich die Aufführungen im Züricher Kreis der Dadaisten, die rasch auch in den Metropolen Lateinamerikas bekannt wurden. Schließlich wurde auch - und zwar gerade aus Paris, das für die Lateinamerikaner noch immer das Zentrum des Fortschritts und aller Sehnsüchte war - der französische Surrealismus rezipiert. Er wirkte mit seiner Aufwertung der dem Bereich des Bewussten entzogenen Strukturen, mit seiner Experimentierfreudigkeit bezüglich neuer Schreibformen - denken wir nur an die „écriture automatique“ - oder auch mit der Grundüberzeugung, dass sich nicht die Kunst am Leben, sondern das Leben an der Kunst zu orientieren habe. 
All dies waren Erfahrungen, welche die Künstler und Schriftsteller in Lateinamerika in grundlegender Weise prägten. So wie das Gründungsmanifest Marinettis wenige Wochen später schon in Bonaerenser Blättern nachzulesen war, fiel auch das erste Manifest des französischen Surrealismus von 1924 in Übersee auf höchst fruchtbaren Boden. An dieser Stelle sei noch eine zweite Anekdote erwähnt, die ein bezeichnendes Licht auf den transatlantischen Dialog und die literarisch-politische Entwicklung der Avantgarden in Lateinamerika wirft. Als die Forderungen nach einer grundlegenden „Reforma universitaria“ in Lateinamerika mit ihrer den gesamten Subkontinent erfassenden Studentenbewegung einsetzten, da waren es sehr wohl avantgardistische Vorstellungen, welche den Studenten auch politischen Rückhalt gaben. Das Grundgerüst ihrer Forderungen aber bildete die Rezeption des berühmtesten Werkes des uruguayischen Modernisten José Enrique Rodó und dessen Wirkung im „Arielismo“. Man könnte von einem Links-Arielismus sprechen, der die Studenten gerade in ihrer Haltung gegenüber den expandierenden USA sehr zustatten kam. Auch bei dieser aufschlussreichen Anekdote deutet sich eine substanzielle Verbindung zwischen Modernisten und Surrealisten an, die keineswegs die These vom Bruch der Avantgardisten mit dem Modernismo und einem Kampf gegen die dominante literarische Tradition zu erhärten in der Lage wäre. In den Ländern Lateinamerikas positionieren sich die historischen Avantgarden anders als in Europa mit Blick auf ihre Beziehung zur literarisch-künstlerischen Tradition.

Frontale Gegenüberstellungen, wie sie in der einschlägigen, auf Lateinamerika bezogenen Avantgarde-Forschungsliteratur über lange Zeit üblich waren, sind bis heute nicht verschwunden, ließen sich aber leicht durch bruchlose intertextuelle Relationen sowie durch Kontinuitäten wie die soeben angesprochenen entkräften. Gleichwohl gilt auch, dass sich die Diskurse und Schreibweisen der Avantgardisten auch in Lateinamerika gegen das Althergebrachte wenden: dominant gewordene Diskursuniversen, denen mit neuen, provozierenden literarischen und lebensweltlichen Adaptationsformen der Garaus gemacht werden soll. Jedoch waren es mehr die offiziellen Diskurse der Macht als die spezifisch literarischen Ausdrucksformen, die im Zielfeuer der avantgardistischen Autoren Lateinamerikas standen.

Harald Wentzlaff-Eggeberts richtige Anmerkung, dass am Ende der Avantgardeforschung in Lateinamerika vielleicht die Einsicht stehen könnte, dass die Avantgardisten die spezifische Suche nach einer eigenen Identität ${ }^{8}$ des Subkontinents vorangetrieben hätten, mag trotz aller Gegenüberstellungen in diese Rich-

8 Vgl. Vives, Ana: Identidad en tiempos de vanguardia. Narcismo, genio y violencia en la obra de Saslvador Dalí y Federico García Lorca. Oxford - Bern: Peter Lang 2015. 
tung einer Kontinuität deuten, waren es doch gerade die Modernisten, die mit aller Dringlichkeit eben diese Frage nach der Identität in den Mittelpunkt rückten. Die ,Identitätsfrage، - dies wird auch unsere Beschäftigung mit Alfonso Reyes' Ifigenia cruel zeigen - ist den Avantgardisten so lieb wie sie den Modernisten teuer war. Vielleicht ist gerade hierin der Kern jener Kontinuität zu erblicken, der sich jenseits veränderter literarischer Formen und Techniken durch die lateinamerikanischen Literaturen zieht und so den Modernismus gleichsam an die grundlegenden Entwicklungen des 20. Jahrhunderts anbindet. Die Bürger'sche These von einer Ästhetik des Bruches kann als Theorie der Avantgarde in Lateinamerika in jedem Falle keine generelle Geltung beanspruchen.

Wann genau begann die Avantgarde in Lateinamerika? Die Antwort auf diese Frage ist gar nicht so leicht! Natürlich lassen sich bereits 1909 die frühe Verbreitung von Filippo Tommaso Marinettis Gründungsmanifest des Futurismus in Buenos Aires und erste literarische Reaktionen hierauf feststellen. Aber es gab einen gewissen zeitlichen Verzug bis zur Herausbildung eigener avantgardistischer Gruppen in der Neuen Welt. Belegen wir dies an einigen Beispielen: Die Minoristen-Gruppe in Kuba formierte sich 1923, vorher schon hatte Borges 1919 bei seiner Rückkehr aus Spanien den spanischen „Ultraísmo“ im Gepäck mitgebracht. Der argentinische Lyriker Oliverio Girondo war nicht weit; und auch der „Creacionismo“ eines Vicente Huidobro siedelte sich schon sehr früh in einem avantgardistischen Kontext an. Vergessen wir nicht 1921 den „Estridentismo“ José Juan Tabladas in Mexiko sowie die Zeitschrift Contemporáneos ebendort, die zwischen 1928 und 1931 - also recht parallel zur kubanischen Revista de Avance, erschien! Viele verschiedene Gruppen, viele Organe, viele Zeitschriften, viele Manifeste, viele Texte wären hier im Grunde $\mathrm{zu}$ besprechen.

Wie sollen Schriftstellerinnen und Schriftsteller, Künstlerinnen und Künstler eingeordnet werden, deren Laufbahn sich zeitweise den Avantgarden annäherte, sie danach aber wieder verließ? Besonders spannend ist der Fall des Argentiniers Jorge Luis Borges, der in Spanien in Berührung mit den historischen Avantgarden kam und dessen erste Gedichtbände unverkennbar im Zeichen dieser Avantgarden standen. Als er aber im Begriff stand, nach grundlegenden ästhetischen Veränderungen und Neuausrichtungen zum späteren ,Vater' der Postmoderne nicht nur in Lateinamerika, sondern weltweit zu werden, fühlte er sich bemüßigt, all seine frühen Bände wieder - sogar eigenhändig - aus den Bibliotheken zu entfernen, also mit seinen Anfängen und mit der historischen Avantgarde zu brechen. Erst in seinen späteren Jahren baute er ein konzilianteres Verhältnis zu seinem Frühwerk auf.

Nahezu in allen lateinamerikanischen Ländern bildeten sich avantgardistische Bewegungen heraus. Allein in Peru lassen sich so verschiedene avantgardistische Entwicklungen aufzeigen wie 1922 César Vallejos Trilce oder Mariáte- 
guis Amauta. Es fällt schwer - und doch ist es notwendig -, all dies dem Label „Avantgarde“ zuzuordnen, handelt es sich doch um so verschiedenartige regionale Ausprägungen und Gedankenwelten, dass bisweilen der innere Zusammenhalt verlorenzugehen drohte. Und doch: Die lateinamerikanischen Avantgardisten sind allesamt bemüht, den europäischen Impulsen nachzugehen, neue, innovative Wege einzuschlagen und so eine Literatur sowie mehr noch öffentlich wirksame Diskurse in ihren Heimatländern modernisierend anzustoßen. Kein Zweifel: Die lateinamerikanischen Avantgardisten sind literarische, ästhetische, künstlerische, politische Neuerer! Aber sind sie auch Umstürzler im literarischen Bereich?

Versuchen wir, die Frage anhand der Analyse konkreter Texte zu beantworten! Dazu eignet sich hervorragend die Behandlung traditioneller Stoffe, klassischer Themen und mehr noch antiker Mythen als Nagelprobe auf die Konsistenz und Provokationslust der lateinamerikanischen Avantgardisten. Fragen wir also nach der Arbeit am Mythos, wie Hans Blumenberg dies ausdrückte! ${ }^{9}$ Auf die literarische Verarbeitung eines anderen Mythos, der Mexikanischen Revolution nämlich, werde ich in unserer Vorlesung später zurückkommen. Dieser Umsturz stellte zwar eine wichtige Epochenerfahrung der Zeitgenossen - und nicht zuletzt der Avantgardisten - dar, doch reicht seine literarische Verarbeitung insbesondere im Roman in wesentlich später zu besprechende Themenstellungen und Bereiche hinein, die freilich nicht vergessen werden sollen.

9 Vgl. Blumenberg, Hans: Arbeit am Mythos. Frankfurt am Main: Suhrkamp 1979. 